\title{
Clinical manifestations and results of cystoscopy in women with interstitial cystitis/bladder pain syndrome
}

\author{
Rashad Sholan \\ Department of Kidney Diseases and Transplantology, Azerbaijan Health Ministry, Republican Centre of Diagnosis and Treatment, Baku, \\ Azerbaijan
}

\begin{abstract}
OBJECTIVE: Interstitial cystitis/bladder pain syndrome (IC/BPS) refers to diseases that are challenging to identify, diagnose and treat. Thus, there is a need to study the clinical and cystoscopic picture of IC/BPS. The present research aims to study the clinical manifestations and results of cystoscopy with hydrodistension in women with IC/BPS.

METHODS: One hundred twenty-six women with clinically diagnosed IC/BPS were examined - their mean age was $46.7 \pm 14.0$ years. Patients were surveyed on pelvic pain and urgency/frequency patient symptom score (PUF), visual analogue scale (VAS) and urgency severity scale (USS). All patients underwent a potassium test (PST) and cystoscopy with hydrodistension. Statistical analysis was performed using SPSS software version 15.0 (SPSS Inc., Chicago, Illinois, USA).

RESULTS: The average PUF score was $8.14 \pm 1.76$ points, VAS $-5.45 \pm 0.93$ points and USS $-2.63 \pm 0.91$ points. A positive potassium test was detected in $91.3 \%$ of cases. The maximum average anatomical capacity of the bladder was $308.0 \pm 77.5$ $\mathrm{ml}$. The maximal cystometric capacity in women with mild pain was higher than among women with moderate and severe pain by $30.9 \%(p<0.05)$ and $53.0 \%(p<0.01)$, respectively. In most cases, mucosal changes were diffuse $(n=57)$ or located in two parts of the bladder. One of the most common symptoms was the diffuse bleeding of the bladder mucosa (III degree). A statistically significant inverse correlation $(r=-0.57, p<0.01)$ was found between the maximal cystometric bladder volume and the severity of the bladder mucosa changes. At the same time, a positive correlation was found between the severity of the bladder mucosa changes and the sum of points on the PUF questionnaire $(r=+0.61, p=0.0003)$, the sum of points on the VAS questionnaire $(r=+0.59, p=0.0008)$ and the USS questionnaire $(r=+0.66, p=0.005)$.

CONCLUSION: A relationship has been established between the clinical manifestations of IC/BPS among examined women and changes in the wall of the bladder. The data obtained from our investigation can help increase IC/BPS diagnostics and improve IC/BPS treatment results.
\end{abstract}

Keywords: Cystoscopy; bladder pain; interstitial cystitis/bladder pain syndrome; women.

Cite this article as: Sholan R. Clinical manifestations and results of cystoscopy in women with interstitial cystitis/bladder pain syndrome. North Clin Istanb 2020;7(5):417-424.

C/BPS refers to diseases that are challenging to iden1 tify, diagnose and treat. According to a RICE study, $2.7 \%$ to $6.5 \%$ of American women have bladder symptoms that are consistent with the IC/BPS diagnosis, indicating the extent of the disease affecting millions of American women $[1,2]$. It is supposed that IC/BPS is more common than it is diagnosed [1-3].
IC/BPS is a chronic debilitating pathological condition that is manifested by pain in the pelvic/suprapubic area and symptoms, such as micturate urge, nycturia, and urinary frequency [3]. Previously, under strict diagnostic criteria, IC/BPS was not diagnosed frequently with an overall prevalence of approximately $0.1 \%$. Currently, due to the wide range of diagnostic opportunities and

Received: December 13, 2019 Accepted: May 15, 2020 Online: August 18, 2020

Correspondence: Rashad SHOLAN, MD. Department of Kidney Diseases and Transplantology, Azerbaijan Health Ministry, Republican Centre of Diagnosis and Treatment, Baku, Azerbaijan.

Tel: +994502125357 e-mail: ittihaf@yahoo.com

(c) Copyright 2020 by Istanbul Provincial Directorate of Health - Available online at www.northclinist.com 
improved understanding of the pathophysiology of the IC/BPS among women, the frequency of the detection of this disease increased, at least to $2 \%$ [2]. However, it is very challenging to diagnose IC/BPS because its manifestations are much similar to many other urological and gynecological diseases $[4,5]$.

IC/BPS has been known for more than 50 years, but the pathophysiology of the disease remains unclear, etiology has not yet been fully studied and diagnosis is based mainly on the exclusion of other diseases [3,5]. It is considered that potential pathophysiological causes of IC/ BPS include a defect in the glycosaminoglycan layer on the apical surface of the bottom of the bladder as well as autoimmune, inflammatory and neurogenic mechanisms and infections $[2,4,5]$.

Diagnosis of IC/BPS is not easy at all. This disease is considered as a "diagnosis of exclusion". Most authors point out the importance of cystoscopy in the diagnosis of IC/BPS in women [6]. It has been reported a relationship between the cystoscopic picture and the duration of the course of the disease [5, 6]. However, data on the relationship between the severity of the clinical manifestations of IC/BPS and changes in the bladder that are detected during cystoscopy are insufficient. It should also be noted the inconsistency of the concepts of diagnosis of IC/BPS. Thus, the National Institute of Diabetes and Digestive and Kidney Diseases (NIDDK) recommends cystoscopic hydrodistension for the diagnosis of IC/BPS and determination of the maximum bladder capacity [7]. However, at the same time, the Society for Urodynamics and Female Urology (SUFU) does not recommend cystoscopic hydrodistension as a test for the diagnosis of IC [8]. According to experts of this society, cystoscopy is necessary when it is clinically necessary [8]. According to the experts of this society, cystoscopy is justified in that case where there is a clinical necessity for it [8]. They explain this by that glomerulation is not specific for the diagnosis of IC/BPS, just as in patients with no symptoms or in patients with other urological or gynecological diseases glomerulation may develop after hydrodistension [8]. At the same time, many researchers have supposed that cystoscopy under anesthesia with hydrodistension in the diagnostic approach of IC/BPS provides an opportunity to objectively classify patients who are often clinically indistinguishable [9]. Thus, there is a need for prospective studies that would clarify the clinical and cystoscopic picture of IC/BPS. All of these data indicate that cystoscopy with hydrodistension is a necessary method and represents the diagnostic tools that are still needed. This study aims to study the clinical manifestations and results of cystoscopy with hydrodistension in women with interstitial cystitis/bladder pain syndrome.

\section{MATERIALS AND METHODS}

One hundred twenty-six women with clinically diagnosed IC/BPS participated in this study. The age of patients ranged from 22 to 76 years and averaged age was $46.7 \pm 14.0$ years. Of these, $52(41.3 \%)$ women were in reproductive age and $74(58.7 \%)$ women were in premenopausal and menopausal ages. The average duration of the disease was $6.0 \pm 2.8$ years. None of the women had signs of urinary infection.

\section{Inclusion Criteria:}

+ women aged 22 years and older;

- the presence of clinical manifestations of IC/BPS lasting at least six months

Exclusion Criteria:

- the presence of acute or exacerbation of a chronic infection of the urinary tract, as well as asymptomatic bacteriuria during the last three months before inclusion in this study;

- the presence of diseases or conditions that could be the cause of pelvic pain and urinary disorders, including neurological diseases, bladder and ureter stones, severe prolapse of the pelvic organs, infravesical obstruction;

- pelvic tumors at present or in history (anamnesis);

- drug treatment for IC/BPS for three months before the start of this study;

- hydrodistension of the bladder and/or a history of the intravesical injections of botulinum toxin;

- negative lidocaine test.

The anatomical capacity of the bladder was determined by ultrasound on an ALOKA scanner (Japan) using a convex multi-frequency sensor with a frequency of 2-4.4 MHz using the standard method.

The IC/BPS diagnosis was based on the NIDDK (The National Institute of Diabetes and Digestive and Kidney diseases) criteria [10]. To assess the intensity of the pain syndrome and the character of the urination disturbances, all patients were questioned according to the scale of the pelvic pain symptoms, imperative frequent urination (pelvic pain and urgency/frequency patient symptom score, PUF). All women underwent a compre- 
hensive examination, which included, in addition to general urological diagnostic methods, a potassium test [11]. This test was carried out in two stages. During the first stage, $40 \mathrm{ml}$ of sterile water was injected into the bladder, after which patients were evaluated for five minutes for pain sense and the appearance of imperative urination. At the second stage, $40 \mathrm{ml}$ of a $0.4 \mathrm{M}$ KCL solution was slowly dripped into the bladder for five minutes. Patients were asked to report their sensation in the bladder during the instillation of the $\mathrm{KCl}$ solution. If a patient-reported discomfort or pain in the bladder during the potassium test, he was asked to assess discomfort (or pain) in the bladder on a 10-point visual analogue scale (VAS). Assessing bladder pain $\geq 3$ or the sudden onset of a sense of urgent urination caused by the test was considered as a positive result. If patients did not feel pain in the bladder but felt a sense of urgent urination during the potassium test, they were asked to assess the severity of the sensation according to the urgency severity scale (USS - urgency severity scale) [12]. The USS questionnaire is a 4-point system and assessed as follows: 0 points - absence of a sense of urgency, 1-2 points - moderate, middle urgency, 3 points - severe urgency, 4 points - inability to hold urine [12]. USS score $\geq 1$ was considered as a potassium test positive. In the event of patients felt pain in the bladder with a VAS score of $<3$ or without urgency, they were considered as having a negative potassium test.

Cystoscopy and hydrodistension were performed in all patients under general anesthesia (intravenous anesthesia). Cystoscopy was carried out by using the endoscopes of the "Olympus" (Japan) company with optics of 30 and 70 degrees in the endoscopic operating conditions. Before performing cystoscopy the condition of the external opening of the urethra and the patency of the urethra were evaluated. During cystoscopy, the location of the ureters' orifices, cystoscopic volume of the bladder, the feature of the optical medium (transparent, with blood, optical medium with suspension) were taken into account and the bladder mucosa (normal, hyperemic, marked hyperemic), the presence of pathological formations (Hunner lesions), submucosal hemorrhage, diffuse submucosal hemorrhage, leukoplakia of the bladder) also were evaluated. When pathological formations were identified, their number and localization (apex, fundus, cervix, right wall, left wall of the bladder) were considered and also the volume of the filled bladder was measured at which these pathological formations were determined. Then, the bladder was emptied and the amount and color of leaked fluid were defined.
Hydrodistension of the bladder was performed according to the following procedure: the bladder was filled with saline until an intravesical pressure reached 80-100 centimeter of the water column; after which the bladder was in stretched condition for two minutes. Then, repeated cystoscopy was performed, the bladder was emptied and the amount of leaked fluid and its color were recorded [13].

This study was conducted in accordance with the principles of the World Medical Association Declaration of Helsinki Ethical Principles for Medical Research Involving Human Subjects [14]. This study was approved by The Local Ethics Committee (Protocol N45 2019. 11-20) and all participants provided their written informed consent.

\section{Statistical Analysis}

Indicators were expressed as a mean of \pm standard deviation (SD) and also as numbers and percentages. The correlation statistical analysis between IC/BPS symptoms and changes in the bladder mucosa was determined by Spearman's rank correlation coefficient. All statistical indicators were bilateral and were considered significant at a p-value of $<0.05$.

\section{RESULTS}

There were no differences between women of different age groups concerning the severity of pain syndrome. According to the PUF questionnaire, mild symptoms appeared in $22(17.5 \%)$ patients, an average degree in 75 (59.5\%) and a severe one in $29(23.0 \%)$ patients. The total score on average was $8.14 \pm 1.76$. The results of the VAS questionnaire showed mild pain (2-4 points) in $25(19.8 \%)$, moderate pain (5-6 points) - in $82(65.1 \%)$ and severe pain (7-8 points) - in 19 (15.1\%) women. The average score was $5.45 \pm 0.93$. Analysis of the results according to the USS scale showed no urgency in five $(4.0 \%)$ patients, moderate average urgency (1-2 points) - in 40 (31.7\%), severe urgency ( 3 points) - in $54(42.8 \%)$ and inability to hold urine ( 4 points) - in $27(21.4 \%)$ patients. The average score, according to the USS scale, was $2.63 \pm 0.91$.

A positive potassium test was detected in $91.3 \%$ of cases, a negative test $-8.7 \%$ of cases. After the administration of potassium, solution pain increased in 100 $(79.4 \%)$ patients. Besides, depending on the symptoms, the frequency of the positive potassium test was different (Fig. 1). According to the results, the potassium test was 


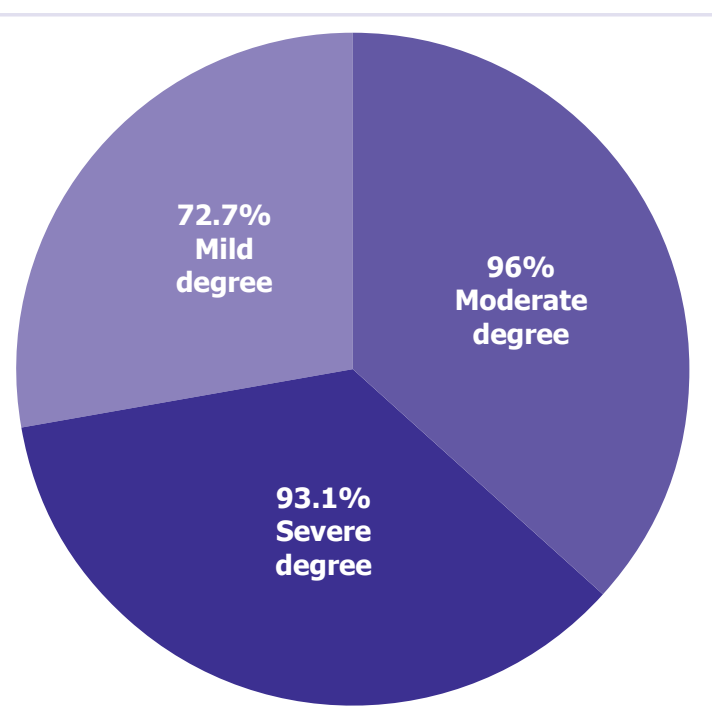

FIGURE 1. The frequency of a positive potassium test, depending on the severity of symptoms (on the PUF scale).

TABLE 1. The severity of urination disorders in women with IC/BPS according to the analysis of urination diaries for three days $(n=126)$

\begin{tabular}{|c|c|c|}
\hline \multirow[t]{2}{*}{ Indicator } & \multicolumn{2}{|c|}{$\begin{array}{c}\text { Urination frequency, } \\
\text { in } 3 \text { days }\end{array}$} \\
\hline & Mean \pm SD & Min.-Max. \\
\hline Number of urination & $33.7 \pm 5.8$ & $24-56$ \\
\hline Number of nightly urination & $8.0 \pm 3.5$ & $0-19$ \\
\hline Urgent urination & $7.1 \pm 3.0$ & $0-28$ \\
\hline The number of nightly urgent urination & $5.3 \pm 2.9$ & $0-20$ \\
\hline The average volume of urination, $\mathrm{ml}$ & $126.2 \pm 22.4$ & $20-260$ \\
\hline
\end{tabular}

SD: Standard deviation; Min.: Minimum; Max.: Maximum.

positive in $16(72.7 \%)$ of 22 patients with a mild degree, in $72(96.0 \%)$ of 75 women with moderate degree, and in $27(93.1 \%)$ of 29 patients with a severe degree.

Most patients noted frequent urination. 112 (88.9\%) women indicated frequent urination more than eight times a day, imperative urination and urge urinary incontinence were reported by $52(41.3 \%)$ and $27(21.4 \%)$ patients, respectively. 25 (19.8\%) women with urgent urinary incontinence had both frequent urination and imperative frequent urination. The nature and severity of urination disorders were revealed according to the urination diaries, which were prepared by women for three days (Table 1). The frequency of urination for three days

\section{TABLE2. The results of cystoscopy in women with IC/BPS}

\begin{tabular}{lcc} 
Bladder mucosa & $\mathrm{n}$ & $\%$ \\
\hline No changes & 20 & 15.9 \\
Hyperemic & 61 & 48.4 \\
Marked hyperemic & 45 & 35.7
\end{tabular}

varied from 24 to 56 (respectively from 8 to 18.7 per day), and averaged $33.8 \pm 5.8$ (respectively 11.2 per day).

We have evaluated the results of cystoscopy at rest and during hydrodistension of the bladder and also compared cystoscopic changes in patients depending on the severity of the clinical manifestations of IC/BPS. Our findings showed that the maximum anatomical capacity of the bladder ranged from 92 to $410 \mathrm{ml}$, which on average, was $308.0 \pm 77.5 \mathrm{ml}$. In 25 patients with mild pain (according to the VAS scale), the value of this indicator ranged from 300 to $390 \mathrm{ml}$, and on average, it was $340.4 \pm 88.6 \mathrm{ml}$. In 82 patients with moderate pain, the maximal anatomical capacity was between the range of $120-280 \mathrm{ml}$ and on average of $235.2 \pm 70.6 \mathrm{ml}$. In 19 women with severe pain, this index was the least value and averaged 160,0 $\pm 48,8 \mathrm{ml}$ ranging between 92-200 $\mathrm{ml}$. Comparative analysis of the maximal cystometric capacity in patients with different pain intensity revealed statistically significant differences. For example, the average maximal cystometric capacity in women with mild pain was higher than in moderate and severe pain by $30.9 \%(p<0.05)$ and $53.0 \%(p<0.01)$, respectively.

At the examination of the external opening of the urethra, in $15(11.9 \%)$ patients, urethral polyps were found. In other cases, the external opening of the urethra was without features. The results of the cystoscopy are presented in Table 2.

During cystoscopy, the focus was on identifying specific pathological changes in the bladder mucosa in IC/ BPS patients. The analysis of the cystoscopic picture was performed using a 5-grade assessment system: 0 no changes in bladder mucosa, I - rare glomerulation, at least in two quadrants, II - diffuse submucosal hemorrhages, III - diffuse mucous bleeding, IV - Hunner lesions. Changes detected during cystoscopy after stretching (hydrodistension) of the bladder ranged from the complete absence of abnormalities to the presence of ulcerative lesions of the bladder mucosa. Most often, 


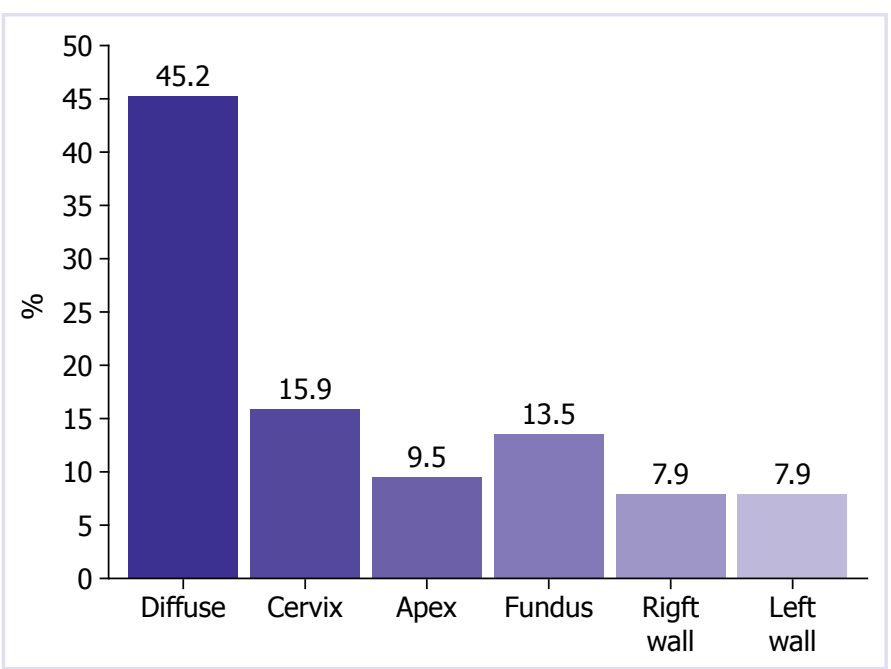

FIGURE 2. Location of the changes in the bladder mucosa according to the cystoscopy in patients with IC/BPS.

TABLE 3. The frequency of changes in the bladder mucosa according to cystoscopy in patients with IC/BPS $(n=126)$

\begin{tabular}{lcc} 
Degree of cystoscopic changes & $\mathrm{n}$ & $\%$ \\
\hline No mucosal changes (0) & 16 & 12.7 \\
Rare glomerulations (I) & 18 & 14.3 \\
Diffuse submucosal hemorrhages (II) & 27 & 21.4 \\
Diffuse bleeding (III) & 49 & 38.9 \\
Hunner lesions (IV) & 16 & 12.7
\end{tabular}

bladder mucosa changes were diffuse $(n=57)$ or located in two sections of the bladder (Fig. 2). Less frequently mucosal changes were detected in the wall of the bladder. Our findings showed that such changes were observed in 10 patients, respectively, in the right and in the left bladder wall.

In this study, 16 women did not have any changes in the mucous membrane of the bladder. The most common feature was diffuse mucosal bleeding (grade III) (Table 3).

The study of correlation relationships revealed a statistically significant inverse correlation $(\mathrm{r}=-0.57, \mathrm{p}<0.01)$ of the maximum cystoscopic volume of the bladder with the severity of changes in the bladder mucosa. A smaller bladder volume correlated with the severity of the changes. A positive correlation was found between the severity of changes in the bladder mucosa and the total score on the PUF questionnaire $(r=+0.61, p=0.0003)$, the total score on the VAS questionnaire $(\mathrm{r}=+0.59, \mathrm{p}=0.0008)$ and total score on the USS questionnaire $(r=+0.66$, $p=0.005)$. There were more severe clinical symptoms in patients with apparent changes in the bladder mucosa. Thus, the results of our studies show that the severity of the clinical symptoms of IC/BPS correlates with the character of the changes in the bladder mucosa.

\section{DISCUSSION}

IC/BPS is a heterogeneous syndrome that includes various phenotypic subgroups of patients with corresponding pathogenetic pathways of the disease. During this investigation, the results of a study of clinical symptoms and cystoscopy with hydrodistension in women with IC/ BPS are presented. The diagnosis of IC/BPS was established on the basis of symptomatic and urological data, including characteristic cystoscopic signs after hydrodistension $[15,16]$. With the help of PUF, VAS, USS questionnaires, we in total assessed the severity of symptoms of dysuria and pain. In IC/BPS, pain and related symptoms are the main problems of this disease [17]. The use of scales, in addition to the symptoms of dysuria, allows you to additionally obtain information about the quality of life of patients.

A potassium test (PST) is also the diagnostic test for IC/BPS. Based on our study, positive PST was detected in 115 (91.3\%) of 126 patients. C.L. Parsons reported about $78 \%$ positivity in patients with clinical IC/BPS [11]. A positive PST indicates a more hypersensitive bladder with pain symptoms. Regarding the sensitivity and specificity of the test, there are opposing views that cast doubt on the clinical value of PST in patients with $\mathrm{BPS} / \mathrm{IC}[16,17]$. We consider that it is necessary to use this test in the diagnosis of IC/BPS. It was found that positive PST provides the diagnosis of urothelium [18]. Urothelium plays a significant role in contact with the nerves of the bladder, smooth muscles, as well as the inflammatory system and cells [18]. Urothelium is a specialized tissue that comprehensively regulates the function of the bladder, and has a secretory and receptor function and plays a fundamental and active role in the pathogenesis of various forms of cystitis and symptoms of the lower urinary tract [19]. Nevertheless, the barrier function of urothelium is the most important of all. The urothelium (the layer of epithelial cells) is located on top of its own plate and covers the inner surface of the bladder. In turn, the urothelium is covered with a glycosaminoglycan layer, which allows minimizing damage to the bladder due to the influence of urine components. To perform this function, urothelium must 
have such properties as urine accumulation and passive permeability. The bladder should be able to accumulate and retain various volumes of urine while maintaining a minimum ratio of urothelium to urine volume. With the exception of actively transported molecules, urothelium should be impervious to all substances in the urine. Moving through the epithelium occurs in two parallel ways - transcellular and through the intercellular space (paracellular pathway). Consequently, the cell membrane and the tight contacts of the urothelium must be impermeable to all components of the urine. Changes in the properties of the cell membrane and/or tight junctions may lead to a change in the barrier function of the urothelium. Damage to urothelium being the main cause of pathology of the bladder causes activation of C-fibers leading to a reduction in smooth muscle, neurogenic inflammation and hypersensitivity of nerve endings. Clinically, this complex of reactions is manifested by frequent imperative urges and chronic pelvic pain - distinctive features of the IC/BPS [18].

Leaking epithelium is considered the main cause that leads to potassium to leak from the urine into the suburothelium and generates the symptoms of urgency and pain in the bladder [18]. Intravesical instillation of $\mathrm{KCl}$ in the concentration of $0.4 \mathrm{M}$ provoked symptoms in $\mathrm{pa}-$ tients with IC and other symptoms of the lower urinary tract $[18,19]$. According to a clinical study by Kuo and Kuo [20], positive PST, pain in the bladder rated more than 2 points on the VAS scale and small bladder capacity were $100 \%$ predictors in patients with IC/BPS.

Performing cystoscopy at rest and with hydrodistension of the bladder revealed characteristic changes: diffuse bleeding in $38.9 \%$ of cases, diffuse submucous hemorrhages in $21.4 \%$ of cases, rare glomerulations in $14.3 \%$ of cases, and Hunner lesions in $12.7 \%$ of cases. Aberrant urothelial cells destroy the permeability barrier; damage to endothelial cells leads to glomerulative bleeding $[21,22]$. It was found that glomerulation during cystoscopic hydrodistension is closely related to overexpression of angiogenic growth factors, such as the growth factor of platelet endothelial cells or thymidine phosphorylase $[23,24]$.

According to the obtained data, a correlation was found between clinical symptoms and changes in the bladder mucosa. In contrast, G.E. Wennevik et al. [25] in peer-reviewed literature found no convincing evidence that glomerulation should be included in the diagnosis or phenotyping of IC/BPS and concluded that glomer- ulation does not correlate with symptoms and are found in patients without IC/BPS.

We observed a more severe clinical course of IC/BPS with a greater degree of severity of changes in the bladder mucosa, which was determined by cystoscopy: diffuse bleeding of the mucosa and Hunner lesions as well as a reduced cystoscopic capacity of the bladder.

The relationship between glomerulation and IC/BPS is currently widely debated. Hunner lesions and glomerulation are believed to be the most characteristic cystoscopic findings in IC/BPS [26]. At the same time, there are reports of the detection of glomerulation in asymptomatic populations [25].

On the recommendation of ESSIC (European Society for the Study of Interstitial Cystitis), cystoscopy should be performed as part of the initial assessment. EAU (European Association Urology), CUA (Canadian Urological Association), SICJ (Society of Interstitial Cystitis of Japan) adhere to the same point of view. At the same time in the recommendations of AUA (American Urological Association) and ICI (International Consultation on Incontinence), cystoscopy is not required as a diagnostic test for IC/BPS but should be performed if another pathological process is suspected $[25,26]$. ICS (International Continence Society) recommended cystoscopy and hydrodistension to detect Hunner lesions and classify them according to the ESSIC classification [27].

Our results confirmed that cystoscopy with hydrodistension in the diagnosis of IC/BPS allows you to objectively classify patients who often do not differ in clinical signs. In this regard, cystoscopy with hydrodistension is important.

Hydrodistension of the bladder during cystoscopy increases the effectiveness of the diagnosis of IC/BPS by increasing the frequency of detection of petechial hemorrhages, as well as ulcerative lesions [15].

In our study, we did not observe complications after hydrodistension. However, there are rare publications in which complications of hydrodistension are noted. At the same time, it is believed that the complications of hydrodistension are not sufficiently appreciated [9].

Bladder hydrodistension is both a diagnostic and a treatment procedure. Ischemic necrosis of the sensory nerves in the cystic wall is considered as possible mechanisms of the therapeutic effects of hydrodistension in IC/BPS, which leads to a reduction in frequent urination, a decrease in the level of heparin-bound epidermal 
factor, which causes a decrease in antiproliferative activity and an increase in microvascularization of the cystic muscle. Different levels of treatment are recommended starting with conservative behavioral procedures, then oral methods of treatment and subsequent intravesical instillations. If these methods are ineffective, a more invasive treatment is suggested, such as botulinum toxin injections, neuromodulation, or surgery. However, there is a scanty database of IC/BPS treatment results. Despite the pathogenetic validity of the use of bladder hydrodistension in the treatment of IC/BPS, there are practically no controlled randomized trials regarding the effectiveness of this treatment method [28].

By analyzing IC/BPS manifestations and bladder conditions in patients with this disease, it is possible to obtain information that will be useful for successful treatment.

\section{Conclusion}

The results of the study showed a relationship between the clinical manifestations of IC/BPS in women and changes in the bladder wall detected by cystoscopy after hydrodistension of the bladder. Patients with a more severe clinical course of the disease revealed more pronounced changes in the bladder. The data obtained can contribute to increasing the efficiency of IC/BPS diagnostics and ultimately improving the results of the treatment of such patients.

Acknowledgements: The author would like to thank all staff of the Department of Kidney Diseases and Transplantology of The Republican Centre of Diagnosis and Treatment.

Informed Consent: Written informed consent was obtained from the patient for the publication of the study.

Ethics Committee Approval: This study was approved by The Local Ethics Committee (Protocol N45 2019-11-20).

Conflict of Interest: No conflict of interest was declared by the author.

Financial Disclosure: The author declared that this study has received no financial support.

\section{REFERENCES}

1. Berry SH, Elliott MN, Suttorp M, Bogart LM, Stoto MA, Eggers P, et al. Prevalence of symptoms of bladder pain syndrome/interstitial cystitis among adult females in the United States. J Urol 2011;186:540-4.

2. Konkle KS, Berry SH, Elliott MN, Hilton L, Suttorp MJ, Clauw DJ, et al. Comparison of an interstitial cystitis/bladder pain syndrome clinical cohort with symptomatic community women from the RAND Interstitial Cystitis Epidemiology study. J Urol 2012;187:508-12. [CrossRef]
3. Fang Z, Xu K. Interstitial cystitis/bladder pain syndrome: A review and an update. Curr Bladder Dysf Rep 2016;11:391-8. [CrossRef]

4. Davis NF, Gnanappiragasam S, Thornhill JA. Interstitial cystitis/painful bladder syndrome: the influence of modern diagnostic criteria on epidemiology and on Internet search activity by the public. Transl Androl Urol 2015;4:506-11.

5. Rosen JM, Klumpp DJ. Mechanisms of pain from urinary tract infection. Int J Urol 2014;21 Suppl 1:26-32. [CrossRef]

6. Cho ST. Is Urethral Pain Syndrome Really Part of Bladder Pain Syndrome? Urogenit Tract Infect 2017;12:22-7. [CrossRef]

7. Hanno PM, Landis JR, Matthews-Cook Y, Kusek J, Nyberg L Jr. The diagnosis of interstitial cystitis revisited: lessons learned from the $\mathrm{Na}$ tional Institutes of Health Interstitial Cystitis Database study. J Urol 1999;161:553-7. [CrossRef]

8. Hanno P, Lin A, Nordling J, Nyberg L, van Ophoven A, Ueda T, et al; Bladder Pain Syndrome Committee of the International Consultation on Incontinence. Bladder Pain Syndrome Committee of the International Consultation on Incontinence. Neurourol Urodyn 2010;29:191-8. [CrossRef]

9. Ens G, Garrido GL. Role of cystoscopy and hydrodistention in the diagnosis of interstitial cystitis/bladder pain syndrome. Transl Androl Urol 2015;4:624-8.

10. Gillenwater JY, Wein AJ. Summary of the National Institute of Arthritis, Diabetes, Digestive and Kidney Diseases Workshop on Interstitial Cystitis, National Institutes of Health, Bethesda, Maryland, August 28-29, 1987. J Urol 1988;140:203-6. [CrossRef]

11. Parsons CL, Greenberger M, Gabal L, Bidair M, Barme G. The role of urinary potassium in the pathogenesis and diagnosis of interstitial cystitis J Urol 1998;159:1862-7. [CrossRef]

12. Chung SD, Liao CH, Chen YC, Kuo HC. Urgency severity scale could predict urodynamic detrusor overactivity in patients with overactive bladder syndrome. Neurourol Urodyn 2011;30:1300-4. [CrossRef]

13. Rigaud J, Delavierre D, Sibert L, Labat JJ. Hydrodistension in the therapeutic management of painful bladder syndrome. Prog Urol 2010;20:1054-9. [CrossRef]

14. World Medical Association Declaration of Helsinki. Ethical Principles for Medical Research Involving Human Subjects. JAMA 2013;310:2191-4. [CrossRef]

15. Cox A, Golda N, Nadeau G, Curtis Nickel J, Carr L, Corcos J, et al. CUA guideline: Diagnosis and treatment of interstitial cystitis/bladder pain syndrome. Can Urol Assoc J 2016;10:E136-55. [CrossRef]

16. Jiang YH, Jhang JF, Kuo HC. Revisiting the Role of Potassium Sensitivity Testing and Cystoscopic Hydrodistention for the Diagnosis of Interstitial Cystitis. PLoS One 2016;11:e0151692. [CrossRef]

17. Hanno PM, Erickson D, Moldwin R, Faraday MM; American Urological Association. Diagnosis and treatment of interstitial cystitis/bladder pain syndrome: AUA guideline amendment. J Urol 2015;193:154553. [CrossRef]

18. Parsons CL. The role of a leaky epithelium and potassium in the generation of bladder symptoms in interstitial cystitis/overactive bladder, urethral syndrome, prostatitis and gynaecological chronic pelvic pain. BJU Int 2011;107:370-5. [CrossRef]

19. Birder LA, Andersson KE, Kanai AJ, Hanna-Mitchell AT, Fry CH. Urothelial mucosal signaling and the overactive bladder-ICI-RS 2013. Neurourol Urodyn 2014;33:597-601. [CrossRef]

20. Kuo YC, Kuo HC. Potential factors that can be used to differentiate between interstitial cystitis/painful bladder syndrome and bladder oversensitivity in women. Int J Clin Pract 2012;66:146-51. [CrossRef]

21. Tamaki M, Saito R, Ogawa O, Yoshimura N, Ueda T. Possible mech- 
anisms inducing glomerulations in interstitial cystitis: relationship between endoscopic findings and expression of angiogenic growth factors. J Urol 2004;172:945-8. [CrossRef]

22. Jhang JF, Kuo HC. Pathomechanism of Interstitial Cystitis/Bladder Pain Syndrome and Mapping the Heterogeneity of Disease. Int Neurourol J 2016;20:S95-104. [CrossRef]

23. Lee JD, Lee MH. Increased expression of hypoxia-inducible factor-1 $\alpha$ and vascular endothelial growth factor associated with glomerulation formation in patients with interstitial cystitis. Urology 2011;78:971. e11-5. [CrossRef]

24. Lee JD, Lee MH. Activation of extrinsic apoptotic pathway from bladder biopsy in patients with interstitial cystitis/painful bladder syn- drome. Urology 2013;82:1451.e7-11. [CrossRef]

25. Wennevik GE, Meijlink JM, Hanno P, Nordling J. The Role of Glomerulations in Bladder Pain Syndrome: A Review. J Urol 2016;195:19-25.

26. Acar Ö, Tarcan T. Cystoscopic evaluation and clinical phenotyping in interstitial cystitis/bladder pain syndrome. J Turk Ger Gynecol Assoc 2019;20:117-22. [CrossRef]

27. Malde S, Palmisani S, Al-Kaisy A, Sahai A. Guideline of guidelines: bladder pain syndrome. BJU Int 2018;122:729-43. [CrossRef]

28. Khullar V, Digesu GA, Veit-Rubin N, Sahai A, Rahnama'i MS, Tarcan $\mathrm{T}$, et al. How can we improve the diagnosis and management of bladder pain syndrome? Part 2:ICI-RS 2018. Neurourol Urodyn 2019;38 Suppl 5:S71-81. [CrossRef] 